\title{
Which Manufacturing Logistics Decisions are Supported by Operational Research? A Literature Survey
}

\author{
Marco Semini ${ }^{1}$, Hakon Fauske ${ }^{2}$, and Jan Ola Strandhagen ${ }^{2}$ \\ 1 Norwegian University of Science, Dept. of Production \& Quality \\ Engineering, NTNU Valgrinda \\ N-7491 Trondheim, Norway \\ 2 SINTEF Technology and Society, Logistics \\ S.P. Andersensvei 5 \\ N-7465 - Trondheim, Norway
}

\begin{abstract}
This paper presents a literature survey of operational research applications to manufacturing logistics decision-making. A total of 80 applications published in Interfaces were reviewed in order to identify the decisions supported, their horizon, and their system boundary. The references to the papers reporting on these applications are included in the paper. Most OR applications were found in short-term production planning and scheduling, plant location and physical distribution system design, production system design, and master planning. Other areas, such as supplier selection and business process design, had few or no applications. While the survey sample does not allow generalizations, the unequal distribution of applications suggests that there is a rather clearly defined set of decision areas that are regularly supported by operational research. Findings also suggest that not only operational, but also certain strategic decisions, such as plant location, plant capacity and equipment capacity, are supported by OR. Finally, a lack of applications crossing organisational boundaries has been identified.
\end{abstract}

\section{Introduction}

Manufacturing logistics deals with the design, planning and control of material flows and related information flows in manufacturing companies and their supply chains. It includes strategic, tactical and operational tasks, with scopes ranging from a single piece of equipment all the way to global supply chains encompassing several independent actors.

Operational research (OR) is concerned with the development and use of quantitative models to support managerial decision-making. It is an umbrella term, covering a wide variety of models and techniques. A number of these, including

Please use the following format when citing this chapter:

Semini, M., Fauske, H. and Strandhagen, J.O., 2008, in IFIP International Federation for Information Processing, Volume 257, Lean Business Systems and Beyond, Tomasz Koch, ed.; (Boston: Springer), pp. 231-238. 
mathematical programming and optimisation, simulation, inventory theory and queuing theory, have been applied frequently to manufacturing logistics decisions, such as network design, production planning and scheduling, and inventory management.

However, OR is based on a number of assumptions limiting its use to a specific kind of situations. In particular, it assumes that the problem situation has been clearly defined and is well structured, that it can be sufficiently well insulated from its wider system, and that it is of a technical nature devoid of politics [1]. Recognizing these limitations, the question arises of where the line is drawn between problem situations that are analyzed by OR, and those that fall outside its scope [2].

The purpose of the survey presented in this paper is to shed some light on this question by reviewing literature reporting on OR applications to manufacturing logistics. All papers published by the journal Interfaces between 1995 and 2004 were surveyed and those describing OR applications in manufacturing logistics selected. The survey's results inform researchers and practitioners about the areas in which OR applications are reported and provide insight about historical trends and future directions for further development. The present paper can also be used in practice and education as a reference to papers reporting on OR applications.

A number of surveys have been carried out previously on the use of OR in industry $[3,4]$. However, recent surveys are mainly concerned with the popularity of different OR techniques, paying little attention to application areas. The lack of recent investigations on OR application areas emphasizes the need for such work.

The remainder of this paper is organised as follows: First, scope and decision areas of manufacturing logistics are presented. Next, the methodological approach is discussed, followed by a presentation and analysis of survey findings. Finally, some conclusions are outlined and future research opportunities suggested.

\section{Manufacturing logistics}

Manufacturing logistics deals with the design, planning and control of material flows and related information flows in manufacturing companies and their supply chains. It includes strategic, tactical and operational tasks, with scopes ranging from a single piece of equipment all the way to global supply chains consisting of several independent actors. Wu et al [5] suggest a taxonomy that characterizes research areas and directions in manufacturing logistics.

Manufacturing logistics encompasses aspects of several overlapping fields, including operations and production management, logistics and supply chain management, and advanced planning. As a consequence, there are several different approaches to structure manufacturing logistics decisions. Chan [6], for example, places the manufacturing planning and control system at the heart of manufacturing logistics. In this paper, a slightly adapted version of the Supply Chain Planning Matrix as defined by Fleischmann et al [7] is employed. 15 decision areas are distinguished and roughly arranged along "decision horizon" and "supply chain process" (Figure 1). The long-term decision areas are shown in a single box to illustrate the comprehensive character of such tasks. Note further that the importance 
and detailed role of each decision area in the matrix varies between enterprises. The matrix is simply one way of organising the decisions constituting the field of manufacturing logistics.

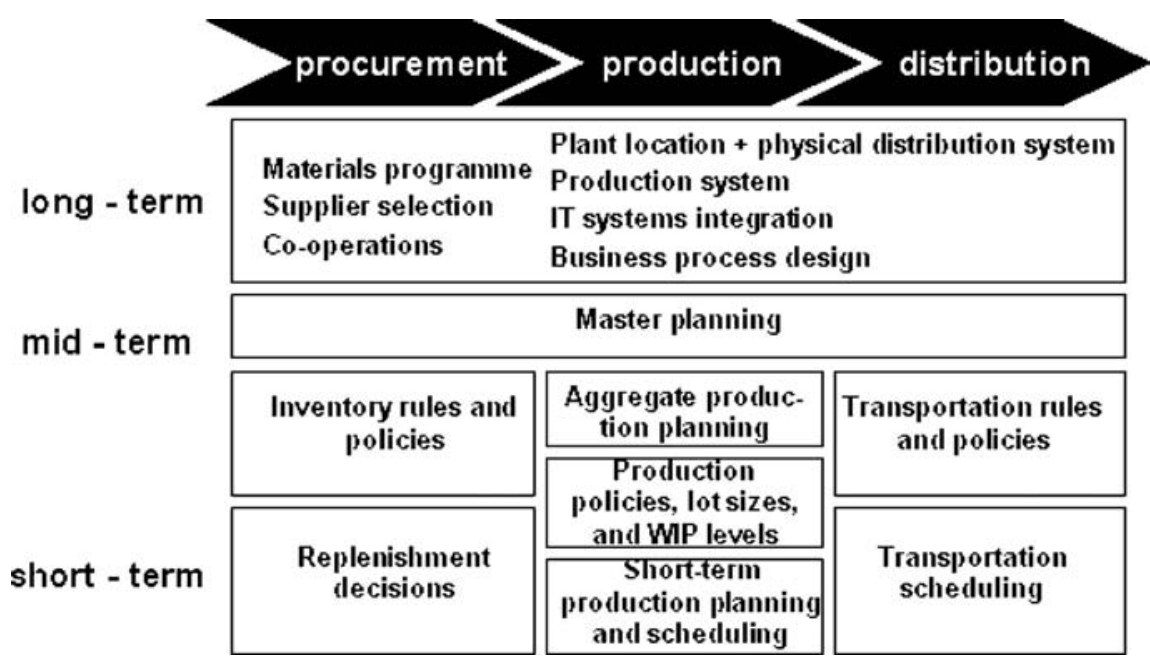

Fig. 1. Manufacturing logistics decision areas (based on Fleischmann et al [7])

In addition to decision horizon and supply chain process, manufacturing logistics decisions can also be arranged along their system boundary (spatial scope). The system boundary separates the system and its environment; the environment may be affected by the decision, but it lies outside its direct control. Wu et al [5] distinguishes the following levels:

- Production Unit - This can be a single piece of equipment, a work station, or a physical grouping of several work stations and equipment such as a manufacturing line or area, a job shop, or a flexible manufacturing cell.

- Production Facility - In a facility, several production units are gathered under one roof and integrated by a certain means, such as vertically by corporate organisation, or horizontally by products.

- Enterprise - This level defines the boundaries of the corporation. An enterprise consists of one or several plants and also includes other aspects of the business, such as design, engineering, marketing and sales.

- Supply Chain - At the broadest level, several independent corporations cooperate to deliver end products. A typical supply chain consists of suppliers, carriers, manufacturers, distributors and warehouses.

\section{Methodology}

The papers included in this study were published by the journal Interfaces between 1995 and 2004. Interfaces is issued by the institute of operations research and the 
management sciences (INFORMS) and entirely dedicated to the documentation of practice and implementation of OR. Between 1995 and 2004, Interfaces published over 500 papers, which were studied one-by-one in order to select those reporting on OR applications that supported manufacturing logistics decision-making in realworld manufacturing companies. This resulted in 60 relevant papers. Some papers describe several applications, resulting in a total of 80 relevant applications included in the analysis.

Since the survey is based on papers from only one journal and from a limited time period, results hold primarily_for Interfaces' publications of the past 10 years. Generalisations to a larger population of OR applications must be made with extreme caution because many OR applications are never published because they have too much or not enough novelty character, because looking only at Interfaces introduces bias due to editorial preferences and the journal's root in the US, and, more generally, because the sample is of very limited size.

Despite these limitation, Interfaces was chosen because it is an important, highquality journal entirely dedicated to the documentation of practice and implementation of OR. The authors are not aware of any other journals of this kind. Interfaces contains numerous articles on real-world applications, all including a detailed description of the decision problem analyzed. A number of other journals occasionally publish application papers, but focus in these journals is on novelty applications. Inclusion of these journals in the study would have biased the results towards novelty applications.

Finally, note that a questionnaire-based survey of companies or practitioners would constitute a useful supplement to the literature survey. The former overcomes most of the limitations of the latter. However, company/practitioner surveys have their limitations as well, including difficulty in acquiring a representative sample, low response rates, problems related to question wording, and incorrectly completed questionnaires. Thus, the present literature survey can constitute one component in a more comprehensive study employing several different research strategies in order to increase validity of results.

\section{Survey findings and analysis}

\subsection{Decision areas}

This survey examined application papers to find out which manufacturing logistics decisions are reported to be supported by quantitative OR techniques. An important finding is the number of applications in each of the specific decision areas introduced in Section 2 (Table 1). It shows that most frequently addressed area is short-term production planning and scheduling (23 applications), followed by plant location and physical distribution system (15 applications), production system (11 applications), and master planning (9 applications). These four areas account for almost $75 \%$ of all applications, leaving relatively few applications to other areas. Six applications concerned inventory rules and policies, five are related to the development/testing of production rules and policies, such as lot sizes and WIP 
levels (for example number and size of KANBAN cards). All other areas had three or fewer applications.

Tabel 1. Number of applications in each decision area of manufacturing logistics

\begin{tabular}{lc}
\hline Decision area & Number \\
\hline Short-term production planning and scheduling & 23 \\
Plant location and physical distribution system & 15 \\
Production system & 11 \\
Master planning & 9 \\
Inventory rules and policies & 6 \\
Production policies, lot sizes and WIP levels & 6 \\
Aggregate production planning & 3 \\
Transportation rules and policies & 2 \\
Transportation scheduling & 2 \\
Materials programme & 1 \\
Supplier selection & 1 \\
Replenishment decisions & 1 \\
Co-operations & 0 \\
IT systems integration & 0 \\
Business process design & 0 \\
\hline
\end{tabular}

While the sample in this study - as mentioned earlier - does not allow generalizations, the unequal distribution suggests that there is a rather clearly defined set of decision areas that are regularly supported by OR. In other decision contexts, different approaches seem to be preferred, such as "soft", qualitative approaches and management accounting. Simple quantitative spreadsheet calculations are also very common in many areas, but such analyses do not normally find their way into research literature. A lack of papers on business process design was not only identified in the present study, but also in a second survey by the authors, which concentrated on applications of one particular OR technique, namely simulation [8]. The findings contradict common claims that business process design is a typical application area of simulation [9].It may well be so that human and organisational aspects are too predominant to warrant an OR-based analysis approach in this and related areas. In conclusion, while many problem areas lie outside the traditional scope of OR implementations, some areas have clearly benefited from such endeavors.

\subsection{Decision horizon}

In the survey, 21 of the decisions supported are of a relatively long-term (strategic) character, 33 are classified as mid-term (tactical), and 26 as short-term (operational). While OR has traditionally been applied mainly in operational decision-making [10], this finding indicates that certain specific long-term decisions, such as plant and equipment capacities, are also analyzed using OR. It rejects claims that OR is not 
suitable for strategic, one-of-a-kind decisions because of their qualitative character and inherent uncertainties [2]. Strategic OR usage has been investigated by numerous authors, including [10].

\subsection{System boundary (spatial scope)}

The spatial scope of the applications in the survey is distributed with 2 applications at supply chain level; 35 at enterprise level; 31 at facility level; and 12 at production unit level. Thus, only two applications cross organisational boundaries, considering supply chains consisting of several independent actors. Similar findings have also been reported in other survey papers $[8,11]$. This is surprising in view of recent years' focus on supply chain management. A possible explanation is that concepts such as supply chain management and global optimization are still relatively new. Only recently, larger parts of supply chains have been analyzed in a holistic way. The novelty character of supply chain simulations may increase the reluctance of industry to reveal the benefits obtained from such studies. Another possible explanation is that logistics decision problems that cross organisational boundaries are characterized by differing strategies and interests, lack of trust and openness, and lacking IT-integration. As a consequence, they may not be easily analysed using quantitative OR techniques. These findings and the explanation are in line with theoretical work on the suitability and limitations of OR; such work emphasizes that in order for OR to be an appropriate approach, high consensus between stakeholders is imperative $[2,12,13]$.

\section{Conclusions and further research}

This paper has presented a literature survey of operational research (OR) applications to manufacturing logistics. The purpose of the survey was to shed light on the question of which manufacturing logistics decisions are supported by OR. Its findings hold primarily for publications in Interfaces from 1995 to 2004, since this constituted the sampling frame in the study. Generalizations should therefore be made with extreme caution. Nevertheless, the survey provides evidence for several conclusions:

- Most OR applications were found in short-term production planning and scheduling, plant location and physical distribution system, production system, and master planning. This indicates that these areas have frequently been analyzed by OR. Other areas, such as business process design, have received little attention; this supports the claim that the scope of OR is limited to specific decision areas; decisions outside these areas can be analyzed using other, "softer" approaches and, amongst others, management accounting.

- Several applications have supported decisions related to plant location, plant capacity, and equipment capacity. This indicates that some long-term strategic decisions are analyzed by OR, in spite of claims that OR is not suitable in such situations. 
- Despite recent years' focus on supply chain management, there have been very few OR applications to supply chains consisting of several independent actors, such as supplier-manufacturer or manufacturer-distributor. This supports theoretical research emphasizing that in order for OR to be an appropriate approach, high consensus between stakeholders is imperative.

This study contributes to a more detailed understanding of the line between decisions analyzed by OR, and those that fall outside its scope. Further research is required to investigate the decision contexts supported by OR. An industry survey could be carried out in order to support or falsify the present study's findings. In addition, empirical and conceptual investigation of the link between decision areas and different OR techniques, such as optimisation or simulation, can provide useful insights for researchers and practitioners.

\section{References}

1. H.G. Daellenbach and D.C. McNickle, Management Science - Decision Making through Systems Thinking (Palgrave, 2005).

2. J. Rosenhead and J. Mingers, in: Rational Analysis for a Problematic World revisited - Problem Structuring Methods for Complexity, Uncertainty and Conflict, edited by J. Rosenhead and J. Mingers (Wiley, Chichester, 2001).

3. C.L. Morgan, A Survey of MS/OR Surveys, Interfaces 19(6), 1989.

4. I. Munro and J. Mingers, The use of multimethodology in practice - results of a survey of practitioners. Journal of the operational research society 53, $369-378$ (2002).

5. S.D. Wu, R.O. Roundy, R.H. Storer and L.A. Martin-VegaWu, Manufacturing logistics research: taxonomy and directions (1997). http://www.lehigh.edu / sdw1/nsfws.pdf

6. J.W.K. Chan, Competitive strategies and manufacturing logistics - an empirical study of Hong Kong manufacturers, Int. J. of Physical Distribution \& Logistics management 35(1), 20-43 (2005).

7. B. Fleischmann, H. Meyr and M. Wagner, in: H. Stadtler and C. Kilger, Supply Chain Management and Advanced Planning ( $3^{\text {rd }}$ edition, Springer, Berlin et al, 2005).

8. M. Semini, H. Fauske and J.O.Strandhagen, Applications of discrete-event simulation to support manufacturing logistics decision-making: a survey. Forthcoming in the Proceedings of the 2006 Winter Simulation Conference, (2006).

9. J. Banks, J.S. Carson, B.L. Nelson, Discrete-event simulation. $2^{\text {nd }}$ edition (Prentice-Hall, Upper Saddle River, 1996). 
10. H. Akkermans and W. Bertrand, On the usability of quantitative modelling in operations strategy decision making, International journal of operations and production management 17(10), (1997).

11. A. Neely, Production/operations management: Research process and content during the 1980s. International Journal of Operations and Production Management 13(1), 1993.

12. A.G. Hopwood, in: Topics in Management Accounting, edited by J. Arnold, B. Carsberg and R. Scapens (Philip Allen, Deddington, 1980).

13. M.C. Jackson and P. Keys, Towards a system of systems methodologies, Journal of the operational research society 35 (1984).

\section{Appendix}

Plant location and physical distribution system: Tyagi et al (34.5); LeBlanc et al (34.2); $2 \mathrm{x}$ Keefer et al (33.2); 2 x Gupta et al (32.4); Keefer et al (32.4); Sery et al (31.3); Linton et al (30.6); Karabakal et al (30.4); Köksalan et al (29.2); Camm et al (27.1); Taube-Netto (26.1); Arntzen et al (25.1); Yoshizaki et al (26.6).

Materials programme: D’Alessandro et al (30.6).

Production System: Srinivasan et al (33.4); Patchong et al (33.1); Gupta et al (32.4); Liberoupoulus et al (32.3); Leachman (32.1); Pfeil et al (30.1); Bermon et al (29.5); Rajaram et al (29.1); Burman et al (28.1); Watson (27.6); Keefer (27.4).

Supplier selection: Wagner et al (26.6)

Master planning: Tyagi et al (34.5); 2 x Gupta et al (32.4); Keefer (32.4); Brown et al (31.6); Schuster et al (28.5); Taube-Netto et al (26.1); Leachman et al (26.1); Arntzen et al (25.1)

Aggregated production planning: Lee et al (32.6); Gazmuri et al (31.4); Taube-Netto et al (26.1)

Inventory rules and policies:; 2 x Bangash et al (34.5); Kapuscinski et al (34.3); Billington et al (34.1); Cohen et al (29.4) Lee et al (25.5)

Production policies, lot sizes and WIP rules: Srinivasan et al (33.4); Denton et al (33.2); Leachman et al (32.1); Tayur (30.5); Vandaele et al (30.1); Rajaram et al (29.1)

Transportation rules and policies: Martin (28.4); Adenso-Diaz et al (28.2)

Replenishments decisions: Katok et al (31.6)

Transportation scheduling: Sesh et al (29.5); Bausch et al (25.2)

Short term production planning and scheduling: Dawande et al (34.3); Keefer (32.5); Gupta et al (32.4); Brown et al (32.3); Leachman et al (32.1); Brown et al (31.6); Katok et al (31.1); Lyon et al (31.1); Olson et al (30.5); Katok et al (30.2); Moss et al (30.2); Vandaele et al (30.1); Bermon et al (29.5); Sesh et al (29,5); Mollaghasemi (28.5) Brinkley et al (28.1); Portougal (27.6); Demeester et al (26.2); Leachman et al (26.1); Taube-Netto et al (26.1); Bowers et al (25.4); Flanders et al (25.2); Sinha et al (25.1). 